\title{
Norovirus: the current focus on a major enteropathogen
}

\author{
Norovírus: o foco atual sobre um importante enteropatógeno \\ Norovirus: el foco actual sobre un importante enteropatógeno
}

Over the past eight years, 70 countries have introduced rotavirus vaccines into their National Immunisation Programs and this has resulted in a rapid and dramatic impact on morbidity and mortality associated with all-cause and rotavirus-related gastroenteritis (GE). In Brazil, one of the worldwide early adopter countries of universal rotavirus vaccination, a significant decline in GE-related hospitalisations and deaths among infants was seen, translated into rates as high as $48 \%$ and $54 \%$, respectively'. While a major reduction in severe rotavirus GE has been experienced by several countries where routine rotavirus vaccine was implemented, norovirus is proportionally (and gradually) becoming the leading cause of severe childhood GE cases that require hospitalisation. The current scenario would therefore highlight the importance of norovirus which, for several decades, has been well recognised as the most important cause of sporadic cases and gastroenteritis outbreaks affecting people of all ages ${ }^{2}$.

In 1972, the late professor Albert Z. Kapikian, from the National Institutes of Health, Bethesda, MD, USA, was the first to identify the so-called Norwalk agent in a stool filtrate derived from an outbreak of acute gastroenteritis in an elementary school in Norwalk, Ohio, USA, which further turned to be the prototype norovirus ${ }^{3}$. The Norwalk agent was a $27-\mathrm{nm}$ virus like particle discovered using the immune electron microscopy (IEM) method that enabled visualisation of virus particles aggregated by convalescent sera from patients who had become ill during the Ohio gastroenteritis outbreak. The use of IEM also enabled the discovery of additional 27-nm gastroenteritis viruses which were then named according to the location they were found, for instance, Hawaii, Montgomery County and Snow Mountain. These viruses were further recognized as belonging to the Norovirus genus, Caliciviridae family.

What appears to be clearly increasing in the current scenario of post-rotavirus vaccine introduction and the worldwide growing accessibility to modern molecular diagnostic techniques is the awareness about the importance of norovirus, not only as the single most significant cause of outbreaks of non-bacterial GE, but also as a major enteropathogen among medically attended children with acute gastroenteritis.

In a recent issue (June 27, 2014) of the Lancet Infectious Diseases, Ahmed and colleagues ${ }^{4}$ reported a meta-analysis of 175 selected articles and more than 187,000 patients included, where the worldwide prevalence of norovirus-related acute GE yielded 18\% (95\% Cl 17-20). Noticeably, unlike rotavirus the prevalence of norovirus was in general higher at community level $[24 \%(95 \% \mathrm{Cl} 18-30)]$ and outpatient settings $[20 \%(95 \% \mathrm{Cl} 16-24)]$, as compared to inpatient environments [17\% (95\% Cl 15-19)]. In addition to the inherent morbidity, whereas mortality due to norovirus disease in the paediatric population is rare in high-income countries, recent estimates point to the occurrence of 200,000 norovirus-related deaths annually among children aged less than 5 years in the least developed countries².

Typically norovirus causes an acute debilitating illness that courses mainly with vomiting, abdominal cramps, fever and watery diarrhoea which may usually last two to three days, although these symptoms may persist for a longer period in hospital outbreaks and among children aged less than 11 years ${ }^{5}$. In general no marked differences in clinical presentation are seen between adults and children, although diarrhoea appears to be a more prominent and frequent symptom than vomiting among adults, whereas children more often develop nausea and vomiting. Elderly people, young children, patients with chronic diseases and immunocompromised individuals are more prone to develop severe disease which may lead to a fatal outcome.

Norovirus is recognised as a highly contagious agent, with fewer than 20 viral particles being required to cause infection. Several sources for transmission have been described including contaminated food or water, contact with an infected person, aerosols and contaminated surfaces. Norovirus is highly stable in the environment, a factor that enhances its potential for spreading either by person-to-person transmission or through contaminated food, particularly in closed and semi-closed communities such as hospital wards, schools, long-term care facilities, prisons and cruise ships ${ }^{5}$. 
Several molecular studies have shown a high degree of norovirus genetic diversity and such a remarkable potential for genome mutability may account for norovirus being currently recognised as the most common cause of viral gastroenteritis worldwide. As based on the sequencing analysis of VP1 (the major capsid protein) noroviruses have been assigned to five distinct genogroups (GI to GV) comprising at least 35 genetic clusters (or genotypes). Genogroups Gl, GIl and GIV are known to infect humans, with genotypes Gll.4 and GI.1 (the prototype strain originally named Norwalk agent) being those more commonly identified ${ }^{6}$. Although numerous genotypes are known to infect humans, over the last decade Gll.4 strains have remained dominant and are currently responsible for the majority of outbreaks that occur worldwide. Moreover, as compared to other genotypes Gll.4 strains are shed in larger quantity during infection, are in general associated with more severe illness and may bind to multiple histo-blood group antigens that are norovirus ligands present on mucosal surface. Since Gll.4 lineage has a higher rate of evolution on average, it is likely that new variants may emerge on a regular basis, possibly every two to three years. In a recent population-based study conducted in a Peruvian periurban community where a birth cohort of children was followed-up during $>3$ years, serial infections caused by the same norovirus genogroup were common ${ }^{7}$. Furthermore, the fact that $97 \%$ of these repeat infections were associated with either distinct genotypes or Gll.4 variants suggests that genotype-specific immunity provides only a low level of cross-protection and that norovirus vaccines should possibly include multiple genotypes in order to induce a broad immunity and achieve clinical protection.

The laboratory diagnosis of norovirus infection may essentially include the detection of viral antigen through ELISA or immune-chromatography, nucleic acid assays, or, less frequently, the use of electron microscopy for visualising virus particles in stool or vomit specimens ${ }^{5}$. Although the ELISA or immune-chromatography can be used for a preliminary identification of norovirus during acute GE outbreaks, negative results should be further confirmed by reverse-transcription polymerase chain reaction (RT-PCR) reference methods. Currently these methods may be available in public health and clinical virology laboratories but are not routinely used in clinics or hospitals. A growing number of clinical virology or research laboratories is performing the quantitative RT-PCR (mainly the TaqMan-based real time assays) to detect norovirus. Genotyping of positive samples can subsequently be performed by DNA sequencing using conventional RT-PCR products.

With regards to the prevention and control, hand hygiene remains as the single most important measure for preventing norovirus infections and controlling transmission. Thorough hand-washing using warm running water and plain soap seems effective in reducing the number of viable viruses via mechanical removal. It remains controversial the efficacy of alcohol-derived and other hand sanitizers for prevention and control of norovirus spread. A second important recommendation toward the prevention and control of norovirus infection is exclusion and isolation of infected persons, due to the highly infectious nature of norovirus; this is particularly important in closed and semi-closed communities. A third method that may effectively interrupt (or minimise) norovirus spread is represented by environmental disinfection, particularly in areas of greatest contamination such as bathrooms, door knobs and hand rails. Sodium hypochlorite solutions (chlorine bleach) have been widely used for environmental disinfection in norovirus outbreaks (e.g., in cruise ships $)^{5}$. All potentially contaminated food must be discarded since norovirus is heat-stable.

There is no specific treatment for norovirus GE and antibiotic therapy is not effective to combat the illness. Most importantly to avoid dehydration is the recommendation of drinking plenty of liquids, particularly if affected persons are young children and the elderly. Mild dehydration may require a visit to an emergency department and in case of severe dehydration hospitalisation is needed for intravenous fluid replacement.

Although a current consensus exists in that a vaccine intervention may be instrumental in achieving a substantial reduction in norovirus disease and in preventing outbreaks, several challenges toward vaccine development remain to be addressed $^{8}$. A key issue that has hampered the development of effective norovirus vaccines is the lack of an in vitro cell culture or small animal model that would allow human norovirus cultivation and assaying infectivity. In addition, a number of other major questions remain to be elucidated such as the duration of immunity, the breadth of protection against the numerous genotype strains, the influence of pre-existing antibodies, and whether protection may be afforded against a broad range of infections, including those which are asymptomatic. A promising strategy toward the development of a norovirus vaccine is based on the use of norovirus-like particles (VLPs) as antigens which have been evaluated in human clinical studies through oral, nasal and intra-muscular routes ${ }^{8}$. These VLPs are non-infectious, because they lack viral RNA, but are immunogenic, since the antigen conformational structure is preserved and mimics interactions with cell receptors. Preliminary results from these studies show that VLP vaccines induce antibodies that block virus binding and confer protection against homologous viral challenge. 
A phase III trial is planned to be conducted with a norovirus, bivalent (GI. 1 and GII.4) VLP vaccine for intra-muscular administration in several settings throughout the world, including Belém, Pará State, Brazil, where epidemiological studies have shown that norovirus may account for as high as 36\% of hospitalisations due to GE among $<5$ years children?.

Even though the overwhelming burden of norovirus disease appears to be more widely recognised in the current post-rotavirus vaccine introduction era, it is likely that a replacement phenomenon is not happening; rather than this, norovirus may be gradually becoming the leading cause of childhood gastroenteritis in relative terms only. Notwithstanding this, the development of a universal, probably multivalent norovirus vaccine is already foreseen, representing a current major goal to be pursued to combat this elsewhere characterised as a possible "perfect human pathogen"10.

\section{REFERENCES}

1 Linhares AC, Justino MC. Rotavirus vaccination in Brazil: effectiveness and health impact seven years post-introduction. Expert Rev Vaccines. 2014 Jan;13(1):43-57.

2 Patel MM, Widdowson MA, Glass RI, Akazawa K, Vinje J, Parashar UD. Systematic literature review of role of noroviruses in sporadic gastroenteritis. Emerg Infect Dis. 2008 Aug; 14(8):1224-31.

3 Kapikian AC. The discovery of the 27-nm Norwalk virus: an historic perspective. J Infect Dis. 2000 May; 181 Suppl 2:S295-302.

4 Ahmed SM, Hall AJ, Robinson AE, Verhoef L, Premkumar P, Parashar UD, et al. Global prevalence of norovirus in cases of gastroenteritis: a systematic review and meta-analysis. Lancet Infect Dis. 2014 Aug;14(8):725-30.

5 Hall AJ, Vinjé J, Lopman B, Park GW, Yen C, Gregoricus N, et al. Updated norovirus outbreak management and disease prevention guidelines. MMWR Recomm Rep. 2011 Mar;60(RR-3):1-18.

6 Hong-ying Z, Li-min S, Wei L, Xuan W, Meng-kai Q, Min H, et al. Molecular epidemiology of genogrup II norovirus infection in outpatients with acute gastroenteritis in Nanging, China (2010-2013). BioMed Res Intern. 2014 Jul; ID 620740:1-7.

7 Saito M, Goel-Apaza S, Espetia S, Velasquez D, Cabrera L, Loli S, et al. Multiple norovirus infections in a birth cohort in a Peruvian periurban community. Clin Infect Dis. 2014 Feb;58(4):483-91.

8 Richardson C, Bargatze RF, Goodwin R, Mendelman PM. Norovirus virus-like particle vaccines for the prevention of acute gastroenteritis. Expert Rev Vaccines. 2013 Feb;12(2):155-67.

9 Siqueira JA, Linhares AC, Carvalho TC, Aragão GC, Oliveira DS, Santos MC, et al. Norovirus infection in children admitted to hospital for acute gastroenteritis in Belém, Pará, Northern Brazil. J Med Virol. 2013 Apr;85(4):737-44.

10 Hall AJ. Noroviruses: the perfect human pathogens? J Infect Dis. 2012 Jun;205(11):1622-4.

Alexandre da Costa Linhares

Editor Associado da Rev Pan-Amaz Saude

Instituto Evandro Chagas/SVS/MS, Ananindeua, Pará, Brasil
Yvone Benchimol Gabbay

Pesquisadora da Seção de Virologia

Instituto Evandro Chagas/SVS/MS, Ananindeua, Pará, Brasil 